\title{
GENERALISATION OF HOOKE'S LAW FOR FINITE STRAIN TO INCLUDE THE ELASTIC RANGE OF STRAIN-HARDENING MATERIALS
}

By

E. W. BILLINGTON

PO Box 111, Eastleigh, Hampshire, SO53 1ZE, England

\begin{abstract}
The representation theorem for isotropic tensor-valued functions of symmetric second-order tensors is considered in the context of two parameters based on the Lode and Fromm parameters. A geometrical representation is established using the concept of a characteristic representation intensity function. It is shown that this geometrical representation identifies the only admissible form of the representation intensity function to be piecewise linear and continuous. This conclusion imposes a restriction on how the representation theorem can be used to formulate constitutive equations. The representation theorem is used to formulate a generalisation of Hooke's law for finite strain that is applicable to the initial elastic range of strain-hardening materials, including the elastic conditions at initial yield.
\end{abstract}

1. Introduction. For any nonlinear constitutive theory to have engineering relevance, it must predict the properties characteristic of the response of typical materials. A distinction must be made between general predictions, which any theoretically admissible constitutive theory must yield, and specific predictions, that is, quantitative predictions that distinguish uniquely between constitutive theories. Prediction of the Poynting [8,9] effect in the context of simple shear is generally cited as evidence for the utility of any proposed nonlinear constitutive theory. Simple equibiaxial extension is effectively a general prediction since any theoretically admissible constitutive equation for isotropic, perfectly elastic materials would be expected to predict stress equality for this simple mode of deformation. The constitutive equation

$$
\mathbf{S}=\lambda(\operatorname{tr} \mathbf{E}) \mathbf{I}+2 G \mathbf{E}
$$

describes in detail the mechanical response of a class of isotropic perfectly elastic solids [1]. In (1.1), $\mathbf{E}$ is the left bi-configuration finite strain tensor and $\mathbf{S}$ is the corresponding stress tensor. For sufficiently small strains the constitutive equation (1.1) reduces to the

Received March 4, 2004.

1991 Mathematics Subject Classification. Primary 74A.

E-mail address: e.billington@ukgateway.net 
stress-strain relationship of the classical infinitesimal linearized theory of elasticity, that is, the generalised Hooke's law. The constitutive equation (1.1) is thus a finite strain form of the generalised Hooke's law and in this context the $\lambda$ and $G$ given in (1.1) are referred to as the generalised Lamé coefficients. The constitutive equation (1.1) admits the general prediction regarding the Poynting effect and the general prediction regarding stress equality for the equibiaxial extension mode of deformation. The quantitative predictions given by the constitutive equation (1.1) are in good accord with the observed mechanical behaviour of various test materials.

The present discussion is concerned with a generalised form of the constitutive equation (1.1) applicable to the initial elastic range of strain-hardening materials, including the elastic conditions at initial yield. Central to the formulation of a general constitutive equation that has (1.1) as a limiting form, is the use of the representation theorem for isotropic tensor-valued functions of symmetric second-order tensors to formulate an appropriate basic constitutive assumption (see for example [2]). The properties of this representation theorem are characterized by three scalar invariant coefficients which are here referred to as the representation coefficients. The question of whether the required basic constitutive assumption can be centred on the use of this representation theorem can only be resolved by deriving expressions for the representation coefficients. Derivation of expressions for the representation coefficients is facilitated by the use of two parameters based on the Lode [5] and Fromm [3] parameters.

2. The Lode and Fromm parameters. Using a prime to denote a deviator, there is associated with the arbitrary symmetric second-order tensor $\mathbf{S}$ and its deviator $\mathbf{S}^{\prime}$ the parameter

$$
\mu=3 s_{1}^{\prime} /\left(s_{3}^{\prime}-s_{2}^{\prime}\right)
$$

where the $s_{i}^{\prime}(i=1,2,3)$ are the eigenvalues of $\mathbf{S}^{\prime}$. The parameter defined in (2.1) was introduced by Lode [5] in the context of continuum plasticity. However, this parameter has general application in the theory of symmetric second-order tensors. In this latter context $\mu$ will be referred to as the Lode parameter for a symmetric second-order tensor, or simply as the Lode parameter. The ordering of the indices in (2.1) is an arbitrary choice. Other admissible forms for the Lode parameter follow from cyclic rotation of the indices in (2.1).

The characteristic equation of $\mathbf{S}^{\prime}$ yields

$$
s_{i}^{\prime}=2\left(\mathrm{~S}_{2}^{\prime} / 3\right)^{1 / 2} \cos \left[(1 / 3) \arccos \omega^{1 / 2}+(2 / 3)(3-i) \pi\right](i=1,2,3)
$$

where

$$
\omega=\frac{27}{4} \frac{S_{3}^{\prime 2}}{S_{2}^{\prime 3}}=\mu^{2} \frac{\left(\mu^{2}-9\right)^{2}}{\left(3+\mu^{2}\right)^{3}}\left(\begin{array}{l}
0 \leqslant \mu^{2} \leqslant 1 \\
0 \leqslant \omega \leqslant 1
\end{array}\right),\left(\begin{array}{l}
1 \leqslant \mu^{2} \leqslant 9 \\
1 \geqslant \omega \geqslant 0
\end{array}\right),\left(\begin{array}{l}
9 \leqslant \mu^{2} \leqslant \infty \\
0 \leqslant \omega \leqslant 1
\end{array}\right)
$$

is the Fromm [3] parameter for a symmetric second-order tensor, and

$$
\begin{gathered}
\mathrm{S}_{2}^{\prime}=\frac{1}{2} \operatorname{tr} \mathbf{S}^{\prime 2}=\frac{1}{3} \mathrm{I}_{\mathbf{S}}^{2}-\mathrm{II}_{\mathbf{S}}=\frac{1}{12}\left(3+\mu^{2}\right)\left(s_{3}^{\prime}-s_{2}^{\prime}\right)^{2} \\
\mathrm{~S}_{3}^{\prime}=\frac{1}{3} \operatorname{tr} \mathbf{S}^{\prime 3}=\frac{2}{27} \mathrm{I}_{\mathbf{S}}^{3}-\frac{1}{3} \mathrm{I}_{\mathbf{S}} \mathrm{II}_{\mathbf{S}}+\mathrm{III}_{\mathbf{S}}=\frac{1}{108} \mu\left(\mu^{2}-9\right)\left(s_{3}^{\prime}-s_{2}^{\prime}\right)^{3}
\end{gathered}
$$


are the second and third principal moments of $\mathbf{S}^{\prime}$ : in (2.4) and (2.5), I $\mathrm{I}_{\mathbf{S}}, \mathrm{II}_{\mathbf{S}}, \mathrm{III}_{\mathbf{S}}$ are the principal invariants of $\mathbf{S}$.

Substitute in (2.1) the expressions for the $s_{i}^{\prime}$ given in (2.2) to give the Lode parameter

$$
\mu=3^{1 / 2} \tan \alpha
$$

where

$$
\alpha=(1 / 6) \arccos (1-2 \omega)=(1 / 6) \arccos \left(1-\left[27 \mathrm{~S}_{3}^{\prime 2} /\left(2 \mathrm{~S}_{2}^{\prime 3}\right)\right]\right) .
$$

Equations (2.6) and (2.7) establish the Lode parameter to be solely a function of the second and third principal moments of $\mathbf{S}^{\prime}$.

3. Mises geometrical representation. The arbitrary symmetric second-order tensor $\mathbf{S}$ and its deviator $\mathbf{S}^{\prime}$ have the spectral representation

$$
\mathbf{S}=\sum_{r=1}^{3} s_{r} \mathbf{q}_{r} \otimes \mathbf{q}_{r} \quad \mathbf{S}^{\prime}=\sum_{r=1}^{3} s_{r}^{\prime} \mathbf{q}_{r} \otimes \mathbf{q}_{r}
$$

where the $s_{i}, s_{i}^{\prime}(i=1,2,3)$ are, respectively, the eigenvalues of $\mathbf{S}$ and its deviator $\mathbf{S}^{\prime}$ and the orthonormal triplet $\mathbf{q}_{i}$ specifies the principal axes common to both $\mathbf{S}$ and its deviator; hence $\mathbf{S}$ and $\mathbf{S}^{\prime}$ are coaxial. Let the principal axes of $\mathbf{S}$ and its deviator $\mathbf{S}^{\prime}$ be orientated so that they are equally inclined to the deviatoric plane through the origin o of the system $(o, q)$. The equation of the deviatoric plane is

$$
\mathrm{S}_{1}^{\prime}=\operatorname{tr} \mathbf{S}^{\prime}=s_{1}^{\prime}+s_{2}^{\prime}+s_{3}^{\prime}=0
$$

where $S_{1}^{\prime}$ is the first principal moment of $\mathbf{S}^{\prime}$. In the deviatoric plane the projected components give the rectangular system of coordinates

$$
\mathrm{u}=\left(s_{3}^{\prime}-s_{2}^{\prime}\right) / 2^{1 / 2} \quad \mathrm{v}=(3 / 2)^{1 / 2} s_{1}^{\prime}
$$

which are common to both $\mathbf{S}$ and $\mathbf{S}^{\prime}$.

Use (3.3) to substitute for the eigenvalues $s_{i}^{\prime}(i=1,2,3)$ in (2.1) to give for the Lode parameter the expression

$$
\mu=3^{1 / 2} \mathrm{v} / \mathrm{u}
$$

The symmetric second-order tensor $\mathbf{S}^{\prime}$ can be represented as a surface $f\left(\mathbf{S}^{\prime}\right)=0$ where $f$ denotes the admissible domain which is a closed convex subset of $\mathbb{R}$ containing the origin o of $\mathbb{R}$ as an interior point. This surface can be set in the three-dimensional eigenspace associated with the eigenvalues $s_{i}^{\prime}$ of $\mathbf{S}^{\prime}$. Use (3.3) to substitute for the eigenvalues $s_{i}^{\prime}$ in the second principal moment of $\mathbf{S}^{\prime}$ given in (2.4) to give

$$
2 \mathrm{~S}_{2}^{\prime}=\mathrm{u}^{2}+\mathrm{v}^{2}=\mathrm{R}^{2}
$$

where use has been made of (3.4). Equation (3.5) is compatible with the characteristic intensity function introduced by Mises $[6,7]$ in the context of plasticity theory: that is

$$
\mathrm{f}=\mathrm{S}_{2}^{\prime}-\left(\mathrm{R}^{2} / 2\right)=0 \text {. }
$$

In the context of (3.6), it follows from (3.5) that the geometrical representation of $\mathbf{S}^{\prime}$, in the form of the characteristic $(\mathrm{u}, \mathrm{v})$ curve in the deviatoric plane, is the Mises circle of radius $\mathrm{R}=\left(2 \mathrm{~S}_{2}^{\prime}\right)^{1 / 2}$ which constitutes a geometrical representation of any symmetric second-order tensor. 
From (3.5), (3.4), and (2.6),

$$
-\mathrm{du} / \mathrm{dv}=\mathrm{v} / \mathrm{u}=\mu / 3^{1 / 2},
$$

which identifies $\mu / 3^{1 / 2}$ as the gradient of the normal to the Mises circle of radius $\mathrm{R}$.

4. Representation theorem. Let $\mathbf{K}$ be a symmetric tensor-valued isotropic function of the arbitrary symmetric second-order tensor $\mathbf{S}$. The representation theorem for isotropic tensor-valued functions of symmetric second-order tensors states that: $\mathbf{K}$ is an isotropic tensor function of $\mathbf{S}$ if and only if

$$
\mathbf{K}(\mathbf{S})=\mathrm{c}_{0} \mathbf{I}+\mathrm{c}_{1} \mathbf{S}+\mathrm{c}_{2} \mathbf{S}^{2}
$$

where the representation coefficients $\mathrm{c}_{0}, \mathrm{c}_{1}, \mathrm{c}_{2}$ are solely scalar-valued functions of the principal invariants $\mathbf{I}_{\mathbf{S}}, \mathrm{II}_{\mathbf{S}}, \mathrm{III}_{\mathbf{S}}$ of $\mathbf{S}$ (see for example [4]).

Let the deviator $\mathbf{K}^{\prime}$ of $\mathbf{K}$ be a symmetric tensor-valued isotropic function of the symmetric second-order tensor $\mathbf{S}^{\prime}$. The representation theorem states that: $\mathbf{K}^{\prime}$ is an isotropic tensor function of $\mathbf{S}^{\prime}$ if and only if

$$
\mathbf{K}^{\prime}\left(\mathbf{S}^{\prime}\right)=\delta_{0} \mathbf{I}+\delta_{1} \mathbf{S}^{\prime}+\delta_{2} \mathbf{S}^{\prime 2}
$$

where the representation coefficients $\delta_{0}, \delta_{1}, \delta_{2}$ are solely scalar-valued functions of the principal moments $\mathrm{S}_{2}^{\prime}, \mathrm{S}_{3}^{\prime}$ of $\mathbf{S}^{\prime}$.

Equation (4.2) also follows from substituting for $\mathbf{K}$ and $\mathbf{S}$ in (4.1) their deviatoric form to give for the representation coefficients the expressions

$$
\delta_{0}=-\frac{2}{3} \mathrm{~S}_{2}^{\prime} \delta_{2}, \quad \delta_{1}=\mathrm{c}_{1}+\frac{2}{3} \mathrm{I}_{\mathbf{S}} \mathrm{c}_{2}, \quad \delta_{2}=\mathrm{c}_{2} .
$$

Enter the expression for $\delta_{0}$ given in (4.3) into (4.2) to give the representation

$$
\mathbf{K}^{\prime}=\delta_{1} \mathbf{S}^{\prime}+\delta_{2}\left(\mathbf{S}^{\prime 2}-\frac{2}{3} \mathbf{S}_{2}^{\prime} \mathbf{I}\right) \text {. }
$$

Equation (4.4) establishes $\delta_{1}$ and $\delta_{2}$ as the only independent representation coefficients.

Although (4.2) follows from (4.1) by substituting for $\mathbf{K}$ and $\mathbf{S}$ their deviatoric form, the representation theorem for $\mathbf{K}^{\prime}$ has been independently stated in (4.2) to establish that $\delta_{1}$ and $\delta_{2}$ are solely functions of the principal moments $\mathrm{S}_{2}^{\prime}, \mathrm{S}_{3}^{\prime}$ of $\mathbf{S}^{\prime}$.

Enter the spectral representations of $\mathbf{S}$ and $\mathbf{S}^{\prime}$ from (3.1) into (4.1) and (4.4), respectively, to give for $\mathbf{K}$ and $\mathbf{K}^{\prime}$ the spectral representations

$$
\mathbf{K}=\sum_{r=1}^{3} k_{r} \mathbf{q}_{r} \otimes \mathbf{q}_{r}, \quad \mathbf{K}^{\prime}=\sum_{r=1}^{3} k_{r}{ }^{\prime} \mathbf{q}_{r} \otimes \mathbf{q}_{r}
$$

In $(4.5)$ the

$$
k_{i}=\mathrm{c}_{0}+\mathrm{c}_{1} s_{i}+\mathrm{c}_{2} s_{i}^{2}, \quad k_{i}^{\prime}=\delta_{1} s_{i}^{\prime}+\delta_{2}\left(s_{i}^{\prime 2}-\frac{2}{3} \mathrm{~S}_{2}^{\prime}\right) \quad(i=1,2,3)
$$

are, respectively, the eigenvalues of $\mathbf{K}$ and $\mathbf{K}^{\prime}$. It is seen from the first equation in (3.1) and the first equation in (4.5) that $\mathbf{S}$ and $\mathbf{K}$ have the orthonormal triplet $\mathbf{q}_{i}$ in common, and hence $\mathbf{K}$ is an isotropic coaxial function of $\mathbf{S}$. Similarly, it follows from the second equation in (3.1) and the second equation in (4.5) that $\mathbf{K}^{\prime}$ is an isotropic coaxial function of $\mathbf{S}^{\prime}$. 
The characteristic equation of $\mathbf{K}^{\prime}$ yields

$$
k_{i}^{\prime}=(2 / 3)^{1 / 2} \mathrm{r} \cos \left[(1 / 3) \arccos (\bar{\omega})^{1 / 2}+(2 / 3)(3-i) \pi\right](i=1,2,3)
$$

where $r=\left(2 K_{2}^{\prime}\right)^{1 / 2}$ is the radius of the Mises circle characteristic of the geometrical representation of $\mathbf{K}$,

$$
\bar{\omega}=\frac{27}{4} \frac{\mathrm{K}_{3}^{\prime 2}}{\mathrm{~K}_{2}^{\prime 3}}=\chi^{2} \frac{\left(\chi^{2}-9\right)^{2}}{\left(3+\chi^{2}\right)^{3}}\left(\begin{array}{l}
0 \leqslant \chi^{2} \leqslant 1 \\
0 \leqslant \bar{\omega} \leqslant 1
\end{array}\right),\left(\begin{array}{l}
1 \leqslant \chi^{2} \leqslant 9 \\
1 \geqslant \bar{\omega} \geqslant 0
\end{array}\right),\left(\begin{array}{l}
9 \leqslant \chi^{2} \leqslant \infty \\
0 \leqslant \bar{\omega} \leqslant 1
\end{array}\right)
$$

is the Fromm [3] representation parameter associated with $\mathbf{K}$ and $\mathbf{K}^{\prime}$, and

$$
\mathrm{K}_{2}^{\prime}=\frac{1}{12}\left(3+\chi^{2}\right)\left(k_{3}^{\prime}-k_{2}^{\prime}\right)^{2}, \quad \mathrm{~K}_{3}^{\prime}=\frac{1}{108} \chi\left(\chi^{2}-9\right)\left(k_{3}^{\prime}-k_{2}^{\prime}\right)^{3}
$$

are the second and third principal moments of $\mathbf{K}^{\prime}$. In (4.8)

$$
\chi=3 k_{1}^{\prime} /\left(k_{3}^{\prime}-k_{2}^{\prime}\right)
$$

is the Lode [5] representation parameter associated with $\mathbf{K}$ and $\mathbf{K}^{\prime}$.

Substitute successively in (4.10) for the $k_{i}^{\prime}$ from the second equation in (4.6) and from (4.7) to give with use of $(3.2)$, together with $(2.1),(2.4)$, and (4.8), the expressions

$$
\chi=\mu\left[1-\frac{3\left(\mu^{2}-1\right)}{2 \mu^{2}} \frac{1}{1-\left(\delta_{1} / \delta_{2} s_{1}^{\prime}\right)}\right]=3^{1 / 2} \tan \phi
$$

where

$$
\phi=(1 / 6) \arccos (1-2 \bar{\omega})=(1 / 6) \arccos \left(1-\left[27 \mathrm{~K}_{3}^{\prime 2} /\left(2 \mathrm{~K}_{2}^{\prime 3}\right)\right]\right) .
$$

Equations (4.11) and (4.12) establish the Lode representation parameter to be solely a function of the second and third principal moments of $\mathbf{K}^{\prime}$.

5. Representation coefficients. Expressions for the representation coefficients follow directly from the representation theorem. However, these expressions have in common a factor which cannot be evaluated from the theorem and recourse must be made to the characteristic representation intensity function for $\mathbf{K}^{\prime}$.

(i) Directly from representation theorem: from the second equation in (4.6)

$$
\begin{aligned}
& \delta_{1}=\left[\frac{\mathrm{H}(3+\chi \mu)^{2}}{\left(3+\chi^{2}\right)\left(3+\mu^{2}\right)}\right]^{1 / 2}\left[1-\frac{\mu\left(\mu^{2}-9\right)(\chi-\mu)}{3\left(\mu^{2}-1\right)(3+\chi \mu)}\right], \\
& \delta_{2}=\left[\frac{\mathrm{H}(3+\chi \mu)^{2}}{\left(3+\chi^{2}\right)\left(3+\mu^{2}\right)}\right]^{1 / 2} \frac{\mu\left(\mu^{2}-9\right)(\chi-\mu)}{3\left(\mu^{2}-1\right)(3+\chi \mu)}\left(\frac{2 \mathrm{~S}_{2}^{\prime}}{3 \mathrm{~S}_{3}^{\prime}}\right)
\end{aligned}
$$

where

$$
\mathrm{H}=\frac{\mathrm{K}_{2}^{\prime}}{\mathrm{S}_{2}^{\prime}}=\left[\delta_{1}+\frac{3 \mathrm{~S}_{3}^{\prime}}{2 \mathrm{~S}_{2}^{\prime}} \delta_{2}\right]^{2}+\frac{\left(4 \mathrm{~S}_{2}^{\prime 3}-27 \mathrm{~S}_{3}^{\prime 2}\right)}{27 \mathrm{~S}_{3}^{\prime 2}}\left[\frac{3 \mathrm{~S}_{3}^{\prime}}{2 \mathrm{~S}_{2}^{\prime}} \delta_{2}\right]^{2}\left(\mathrm{~K}_{2}^{\prime}=\mathrm{HS}_{2}^{\prime}\right)
$$

and use has been made of (2.3), (2.4), (2.5), (4.6), (4.9), and (4.11).

(ii) From characteristic representation intensity function: the counterpart for $\mathbf{K}$ to the intensity function defined for $\mathbf{S}$ in (3.6) is

$$
\mathrm{h}=\mathrm{K}_{2}^{\prime}-\mathrm{k}^{2}=\mathrm{S}_{2}^{\prime} \mathrm{H}\left(\mathrm{S}_{2}^{\prime}, \mathrm{S}_{3}^{\prime}\right)-\mathrm{k}^{2}=0, \quad(\mathrm{k}=\text { const. })
$$


where use has been made of the second equation in (5.3). The characteristic representation intensity function defined on the right-hand side of (5.4) was introduced by Prager [10] in the context of plasticity theory. Since H finds application in the context of the representation theorem it will here be referred to as the Prager representation parameter.

Use the relations

$$
\partial \mathrm{S}_{2}^{\prime} / \partial \mathbf{S}=\mathbf{S}^{\prime} \quad \partial \mathrm{S}_{3}^{\prime} / \partial \mathbf{S}=\mathbf{S}^{\prime 2}-\frac{2}{3} \mathrm{~S}_{2}^{\prime} \mathbf{I}
$$

to rearrange (4.4) to give

$$
\mathbf{K}^{\prime}=\delta_{1}\left(\partial \mathrm{S}_{2}^{\prime} / \partial \mathbf{S}\right)+\delta_{2}\left(\partial \mathrm{S}_{3}^{\prime} / \partial \mathbf{S}\right)
$$

Since the representation coefficients are each solely a function of the principal moments $\mathrm{S}_{2}^{\prime}, \mathrm{S}_{3}^{\prime}$ of $\mathbf{S}^{\prime}$ in accord with the defining statement accompanying the formulation of the theorem given in (4.2), it follows from (5.3) that $\mathrm{H}$ is solely a function of $\mathrm{S}_{2}^{\prime}$, $\mathrm{S}_{3}^{\prime}$. This conclusion implies that the characteristic representation intensity function defined in (5.4) is a function of the principal moments of $\mathbf{S}^{\prime}$ : that is, $\mathrm{h}=\mathrm{h}\left(\mathrm{S}_{2}^{\prime} . \mathrm{S}_{3}^{\prime}\right)$ and hence

$$
\partial \mathrm{h} / \partial \mathbf{S}=\left(\partial \mathrm{h} / \partial \mathrm{S}_{2}^{\prime}\right)\left(\partial \mathrm{S}_{2}^{\prime} / \partial \mathbf{S}\right)+\left(\partial \mathrm{h} / \partial \mathrm{S}_{3}^{\prime}\right)\left(\partial \mathrm{S}_{3}^{\prime} / \partial \mathbf{S}\right) \text {. }
$$

From (5.5) and (5.6)

$$
\mathbf{K}^{\prime}=\partial \mathrm{h} / \partial \mathbf{S} . \quad \delta_{1}=\partial \mathrm{h} / \partial \mathrm{S}_{2}^{\prime}, \quad \delta_{2}=\partial \mathrm{h} / \partial \mathrm{S}_{3}^{\prime} .
$$

Enter the relation for $h$ given on the right-hand side in (5.4) into the second and third equations in (5.7) and use (2.3) to give for the representation coefficients the expressions

$$
\delta_{1}=\mathrm{H}-3 \omega \frac{\partial \mathrm{H}}{\partial \mu} \frac{\partial \mu}{\partial \omega} \quad \delta_{2}=3 \omega \frac{\partial \mathrm{H}}{\partial \mu} \frac{\partial \mu}{\partial \omega}\left(\frac{2 \mathrm{~S}_{2}^{\prime}}{3 \mathrm{~S}_{3}^{\prime}}\right) .
$$

The equality between the expressions $\delta_{1}+\left(3 S_{3}^{\prime} / 2 S_{2}^{\prime}\right) \delta_{2}$ formed from (5.1) and (5.2). and independently from (5.8), gives the Prager representation parameter

$$
\begin{aligned}
\mathrm{H} & =(3+\chi \mu)^{2} /\left[\left(3+\chi^{2}\right)\left(3+\mu^{2}\right)\right] \\
& =\cos ^{2}[(1 / 6) \arccos (1-2 \omega)-(1 / 6) \arccos (1-2 \bar{\omega})], \quad \mathrm{H}:[0,1]
\end{aligned}
$$

where $\mu$ and $\chi$ have been replaced in the first expression for $\mathrm{H}$ in (5.9) from (2.6), (2.7), (4.11), and (4.12) to yield the second expression for $\mathrm{H}$ given in (5.9).

From the first expression for the Prager representation parameter given in (5.9)

$$
\frac{\partial \chi}{\partial \mu}=\frac{\left(3+\chi^{2}\right)}{\left(3+\mu^{2}\right)}\left[1-\frac{\left(3+\mu^{2}\right)}{2[3 \mathrm{H}(1-\mathrm{H})]^{1 / 2}} \frac{\partial \mathrm{H}}{\partial \mu}\right] .
$$

Substitute on the left-hand side of the second equation in (5.8) the expression for $\delta_{2}$ given in (5.2) to yicld

$$
\partial \mathrm{H} / \partial \mu=2[3 \mathrm{H}(1-\mathrm{H})]^{1 / 2} /\left(3+\mu^{2}\right)
$$

where use has been made of (2.3) and the first equation in (5.9). Enter the expression for $\partial \mathrm{H} / \partial \mu$ given in (5.11) into (5.10) to give

$$
\partial_{\chi} / \partial \mu=0 \text {. }
$$

Equation (5.12) establishes that the Lode representation parameter is not a function of the continuously variable Lode parameter. This conclusion. together with the condition 
that the representation coefficients are each solely a function of the principal moments of $\mathbf{S}^{\prime}$ in accord with the defining statement accompanying the formulation of the theorem given in (4.2), requires the Lode representation parameter to be a constant. This implies, with use of (4.8), that

$$
\chi=\text { const. }=\chi_{0}, \chi_{1}, \chi_{2}, \quad \chi_{0}^{2}:[0,1], \quad \chi_{1}^{2}:[1,9], \quad \chi_{2}^{2}:[9, \infty] .
$$

Expressions for the representation coefficients are obtained by entering into (5.1) and (5.2) the expression for the Prager representation parameter given in the first equation in $(5.9)$ : thus

$$
\begin{aligned}
& \delta_{1}=\frac{(3+\chi \mu)^{2}}{\left(3+\chi^{2}\right)\left(3+\mu^{2}\right)}\left[1-\frac{\mu\left(\mu^{2}-9\right)(\chi-\mu)}{3\left(\mu^{2}-1\right)(3+\chi \mu)}\right] \quad(\chi=\text { const. }), \\
& \delta_{2}=\frac{\mu\left(\mu^{2}-9\right)(\chi-\mu)(3+\chi \mu)}{3\left(\mu^{2}-1\right)\left(3+\chi^{2}\right)\left(3+\mu^{2}\right)}\left(\frac{2 S_{2}^{\prime}}{3 S_{3}^{\prime}}\right) \quad(\chi=\text { const. })
\end{aligned}
$$

which also follow from (5.8) with use of (2.3) and the first equation in (5.9).

The condition $\mu=\chi$ reduces $(5.14)$ and (5.15) to

$$
\left[\delta_{1}\right]_{\mu=\chi}=1, \quad\left[\delta_{2}\right]_{\mu=\chi}=0 .
$$

Enter the conditions given in (5.16) into (4.4) and (4.1) to give, with use of (4.3), the conditions

$$
\left[\mathbf{K}^{\prime}\right]_{\mu=\chi}=\mathbf{S}^{\prime}, \quad[\mathbf{K}]_{\mu=\chi}=\mathrm{c}_{0} \mathbf{I}+\mathbf{S}
$$

6. Geometrical representation in the deviatoric plane. There are two geometrical representations in the deviatoric plane. These are the Mises geometrical representation, which centres on the use of the eigenvalues $k_{i}$ of $\mathbf{K}$, and the alternative geometrical representation which, since $\mathbf{K}$ is an isotropic coaxial function of $\mathbf{S}$, centres on the use of the eigenvalues $s_{i}$ of $\mathbf{S}$. The latter gcometrical representation of an isotropic tensorvalued function of a symmetric second-order tensor follows from (5.4) with $\mathrm{H}$ identified as the Prager representation parameter defined in (5.9) and hence it will be referred to as the Prager geometrical representation.

(i) Mises geometrical representation: a geometrical representation of the symmetric second-order tensor $\mathbf{K}$ parallels that for $\mathbf{S}$ given in Section 3. This representation in the deviatoric plane centres on the use of the eigenvalues of $\mathbf{K}$ and is characterised by the Mises representation circle of radius $r=\left(2 K_{2}^{\prime}\right)^{1 / 2}$. The gradient of the normal to the Mises representation circle of radius $\mathrm{r}$ is $\chi / 3^{1 / 2}$. In the context of the Mises representation circle of radius $r$, the Lode representation parameter $\chi$ is continuously variable.

(ii) The Prager geometrical representation: let the principal axes of $\mathbf{S}$ be orientated so that they are equally inclined to the deviatoric plane introduced in Section 3. In (2.1) set

$$
\left(s_{3}^{\prime}-s_{2}^{\prime}\right)=\left.2^{1 / 2} \mathrm{u}\right|_{\mathbf{K}} \quad s_{1}^{\prime}=\left.(2 / 3)^{1 / 2} \mathrm{v}\right|_{\mathbf{K}}
$$

to give with use of $(2.6)$

$$
\mu=\left.3^{1 / 2}(\mathrm{v} / \mathrm{u})\right|_{\mathbf{K}}=\left.3^{1 / 2} \tan \alpha\right|_{\mathbf{K}} .
$$


To distinguish between (6.1), (6.2) and equations (2.6), (3.3), and (3.7), the subscript $\mathbf{K}$ is used to identify $(6.1)$ and (6.2) with the representation theorem.

Substitute the expression for the Prager representation parameter given in the first equation in (5.9) into (5.4) and in the resulting expression enter the relation for $\mathrm{S}_{2}^{\prime}$ given in (3.5) and use (3.4) to give the characteristic representation intensity function

$$
\mathrm{h}=\frac{\left(3^{1 / 2} \mathrm{u}+\chi \mathrm{v}\right)^{2}}{2\left(3+\chi^{2}\right)}-\mathrm{k}^{2}=0 .
$$

Equation (6.3) gives the expression

$$
\mathrm{v}=3^{1 / 2}\left(\mathrm{u}_{0}-\mathrm{u}\right) / \chi, \quad \mathrm{u}_{0}=\mathrm{k}\left[2\left(3+\chi^{2}\right) / 3\right]^{1 / 2}=\text { const. }
$$

The condition given in (5.13) establishes that the characteristic geometrical representation, in the form of the (u, v) relation given in the first equation in (6.4), is linear in the deviatoric plane. It also follows from (2.3) to (2.7) and (4.8) to (4.12), together with $(5.13)$, that this $(\mathrm{u}, \mathrm{v})$ relation is linear in each of the twelve $\pi / 6$ segments in the deviatoric plane. Equations (4.11), (5.13), and the first equation in (6.4) give

$$
\chi=-\left.3^{1 / 2}(\mathrm{du} / \mathrm{dv})\right|_{\mathbf{K}}=\left.3^{1 / 2} \tan \phi\right|_{\mathbf{K}}=\text { const. }
$$

In (6.5), $\chi / 3^{1 / 2}$ is the gradient of any arbitrary straight line perpendicular to the linear (11. v) relation given in the first equation in (6.4).

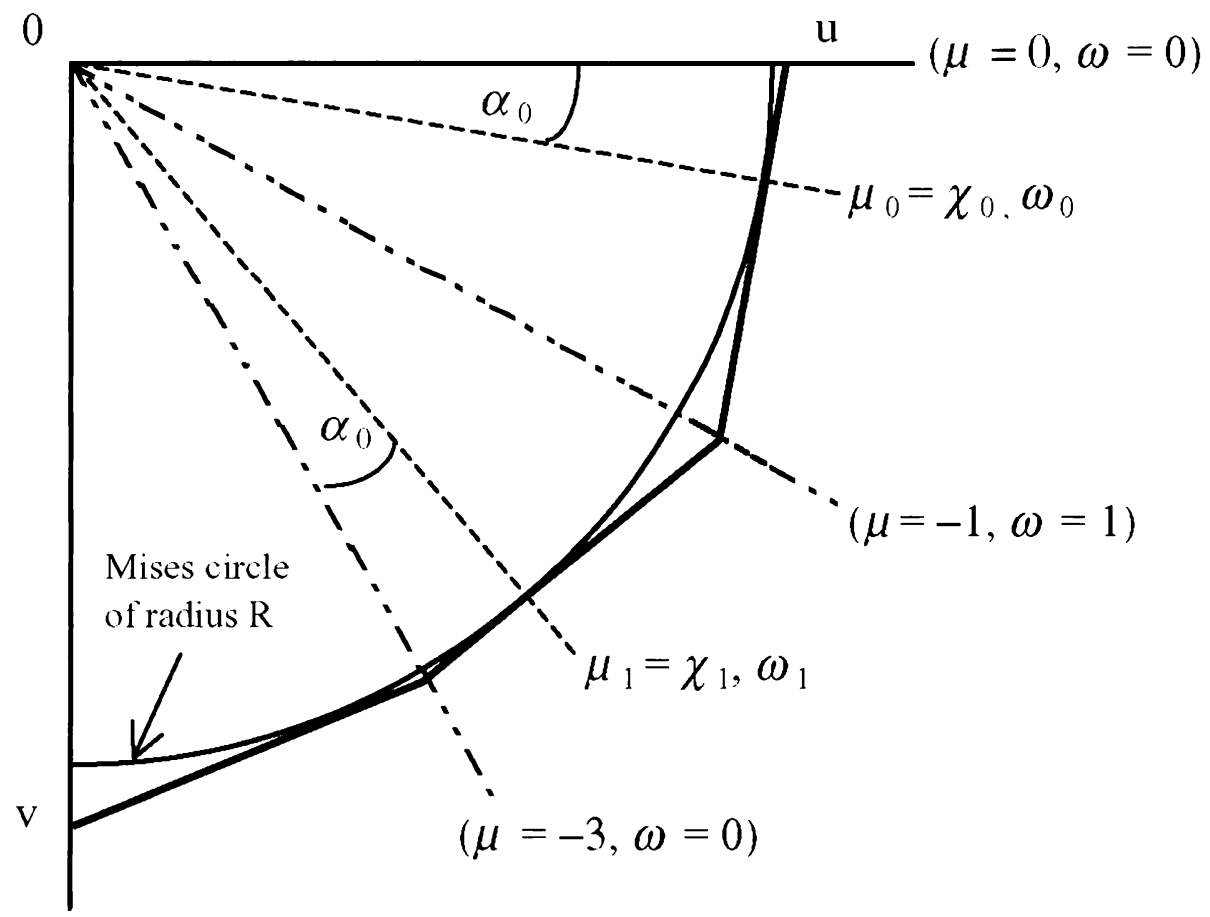

FIc. 1. Variation of $\mathrm{u}$ with $\mathrm{v}$ for $\chi=0.333$ and $\mu:[-\infty, 0]$. 
The geometrical representation of $\mathbf{S}$ in the deviatoric plane is the Mises circle of radius $\mathrm{R}=\left(2 \mathrm{~S}_{2}^{\prime}\right)^{1 / 2}$. For $\mu=\chi$, the first equation in (5.9), together with (5.3), (3.5), (6.5), and (2.6), yield the conditions

$$
[\mathrm{H}]_{\mu=\chi}=1, \quad\left[\mathrm{~K}_{2}^{\prime}\right]_{\mu=\chi}=\mathrm{S}_{2}^{\prime}=\mathrm{R}^{2} / 2, \quad\left[\left.\phi\right|_{\mathbf{K}}=\left.\alpha\right|_{\mathbf{K}}\right]_{\mu=\chi}
$$

from which it follows that for $\mu=\chi$, the line defined by the linear $(\mathrm{u}, \mathrm{v})$ relation given in the first equation in (6.4) is a tangent to the Mises circle of radius $\mathrm{R}$. This condition also holds in all twelve $\pi / 6$ segments in the deviatoric plane. Equation (5.13) identifies two adjacent $\pi / 6$ sections for $\mu=-1$ characterised by $\chi_{0}$ for $\mu:[-1,0]$ and by $\chi_{1}$ for $\mu:[-3$. -1]. The conditions $s_{1}=0, s_{2}=0$ for all $s_{3} \geqslant 0$ when entered into (2.1) give $\mu=-1$. Set $s_{3}=\mathrm{Y}$ and substitute $\mu=-1, s_{1}=0, s_{2}=0, s_{3}=\mathrm{Y}$ into (6.3) to give

$$
\mathrm{Y}=\frac{2\left[3\left(3+\chi_{0}^{2}\right)\right]^{1 / 2}}{\left(3-\chi_{0}\right)} \mathrm{k}=\frac{2\left[3\left(3+\chi_{1}^{2}\right)\right]^{1 / 2}}{\left(3-\chi_{1}\right)} \mathrm{k}, \quad(\mu=-1) .
$$

Equation (6.7) yields

$$
\left(3+\chi_{0}+\chi_{1}-\chi_{0} \chi_{1}\right)\left(\chi_{1}-\chi_{0}\right)=0
$$

and since $\chi_{1} \neq \chi_{0},(6.8)$ gives the condition

$$
\chi_{1}=\left(3+\chi_{0}\right) /\left(\chi_{0}-1\right) .
$$

For the two adjacent $\pi / 6$ sections there are values of the Lode parameter $\mu_{0}=\chi_{0}$, $\mu_{1}=\chi_{1}$ at which each linear section is tangent to the Mises circle of radius $\mathrm{R}=\left(2 \mathrm{~S}_{2}^{\prime}\right)^{1 / 2}$ (see Fig. 1). Hence, for the two points of contact for which $\mu_{0}=\chi_{0}$ and $\mu_{1}=\chi_{1}$, it follows from (6.9) that

$$
\mu_{1}=\left(3+\mu_{0}\right) /\left(\mu_{0}-1\right) .
$$

Denote by $\omega_{0}$ and $\omega_{1}$ the values of the Fromm parameter for $\mu_{0}=\chi_{0}$ and $\mu_{1}=\chi_{1}$ respectively. The result of substituting the expression for $\mu_{1}$ given in (6.10) into the expression for $\omega$ given in (2.3) establishes that

$$
\left.\omega_{0}\right|_{\mu_{0}=\chi_{0}}=\left.\omega_{1}\right|_{\mu_{1}=\chi_{1}} .
$$

From (2.6), $\mu_{0}=-3^{1 / 2} \tan \alpha_{0}$ for $\mu_{0}=\chi_{0}$. Substitute this expression for $\mu_{0}$ into (6.10) to give $\mu_{1}=-3^{1 / 2} \tan \left(\pi / 3-\alpha_{0}\right)$ for $\mu_{1}=\chi_{1}$. These expressions for $\mu_{0}$ and $\mu_{1}$, together with the condition given in (6.11) establish that the segments of the Mises circle of radius $\mathrm{R}=\left(2 \mathrm{~S}_{2}^{\prime}\right)^{1 / 2}$ and the associated tangents in the form of the linear sections given in (6.4) for each of the two adjacent $\pi / 6$ sections are mirror images of each other (see Fig. 1). Hence the two linear sections meet in a vertex for which $\mu=-1$. The same conclusion holds for all the linear sections. From this it follows that the twelve linear segments form a twelve-sided regular polygon, the vertices being identified from (2.3) by the values of the Lode parameter $\mu=0, \pm 1, \pm 3, \pm \infty$, and hence the locus in the deviatoric plane is symmetric with respect to each of the three projected axes. These conclusions are in accord with the condition that $\mathbf{K}$ is a symmetric, tensor-valued isotropic function of the symmetric second-order tensor $\mathbf{S}$. This class of intensity function $\mathrm{h}$ is piecewise linear and continuous and is not continuously differentiable.

The Prager geometrical representation implies that the characteristic representation intensity function is a surface $\mathrm{h}\left(\mathrm{S}_{2}^{\prime}, \mathrm{S}_{3}^{\prime}\right)=0$ in the three-dimensional eigenspace associated 
with the eigenvalues $s_{i}^{\prime}$ of $\mathbf{S}^{\prime}$, where h denotes the admissible representation domain which is a closed convex polyhedron containing again the origin o of $\mathbb{R}$ as an interior point. This conclusion identifies the characteristic representation intensity function as a criterion for determining admissible systems of representation: this interpretation of $h$ is central to the formulation of constitutive equations.

7. Basic constitutive assumption. Let $\mathbf{M}$ be a symmetric tensor-valued isotropic function of the symmetric second-order tensor $\mathbf{E}$. The properties of $\mathbf{M}$ and $\mathbf{E}$ are formally identical to those of $\mathbf{K}$ and $\mathbf{S}$ respectively. Hence, from (4.1)

$$
\mathbf{M}(\mathbf{E})=\mathrm{a}_{0} \mathbf{I}+\mathrm{a}_{1} \mathbf{E}+\mathrm{a}_{2} \mathbf{E}^{2}
$$

where $\mathrm{a}_{0}, \mathrm{a}_{1}, \mathrm{a}_{2}$ are solely scalar-valued functions of the principal invariants $\mathrm{I}_{\mathbf{E}}, \mathrm{II}_{\mathbf{E}}, \mathrm{III}_{\mathbf{E}}$ of E. Equation (7.1) can be expressed in deviatoric form:

$$
\mathbf{M}^{\prime}\left(\mathbf{E}^{\prime}\right)=\varphi_{1} \mathbf{E}^{\prime}+\varphi_{2}\left(\mathbf{E}^{\prime 2}-\frac{2}{3} \mathbf{E}_{2}^{\prime} \mathbf{I}\right)
$$

In (7.2) the response coefficients

$$
\varphi_{1}=\mathrm{a}_{1}+\frac{2}{3} \mathrm{I}_{\mathbf{E}} \mathrm{a}_{2}=\Psi\left[1-\frac{\nu\left(\nu^{2}-9\right)(\kappa-\nu)}{3\left(\nu^{2}-1\right)(3+\kappa \nu)}\right], \varphi_{2}=\mathrm{a}_{2}=\left(\Psi-\varphi_{1}\right)\left(\frac{2 \mathrm{E}_{2}^{\prime}}{3 \mathrm{E}_{3}^{\prime}}\right)
$$

where the Prager response parameter

$$
\Psi=\frac{(3+\kappa \nu)^{2}}{\left(3+\kappa^{2}\right)\left(3+\nu^{2}\right)}, \quad(\kappa=\text { const. })
$$

and

$$
\kappa=\frac{3 m_{1}^{\prime}}{m_{3}^{\prime}-m_{2}^{\prime}}=\text { const. }, \quad \nu=\frac{3 \varepsilon_{1}^{\prime}}{\varepsilon_{3}^{\prime}-\varepsilon_{2}^{\prime}},
$$

are the Lode parameters associated with $\mathbf{M}^{\prime}$ and with $\mathbf{E}^{\prime}$ respectively. In (7.5), the $m_{i}^{\prime}, \varepsilon_{i}^{\prime}(i=1,2,3)$ are, respectively, the eigenvalues of $\mathbf{M}^{\prime}$ and $\mathbf{E}^{\prime}$. The second principal moments of $\mathbf{M}^{\prime}$ and $\mathbf{E}^{\prime}$ are

$$
\mathrm{M}_{2}^{\prime}=\frac{1}{12}\left(3+\kappa^{2}\right)\left(m_{3}^{\prime}-m_{2}^{\prime}\right)^{2} \quad \mathrm{E}_{2}^{\prime}=\frac{1}{12}\left(3+\nu^{2}\right)\left(\varepsilon_{3}^{\prime}-\varepsilon_{2}^{\prime}\right)^{2}
$$

Equations (7.3) to (7.6) are the counterpart to those for $\mathbf{K}^{\prime}$ and $\mathbf{S}^{\prime}$ given in (5.14), (5.15), (5.9), (4.10), (2.4), (4.9), and (2.1).

The constitutive assumption takes the form

$$
\mathbf{K}=2 G \mathbf{P}(\mathbf{M})
$$

where the nonlinear response of $\mathbf{M}$ to changes in the loading of $\mathbf{K}$ is described by the coefficient $G$ through its dependence upon the principal invariants $\mathrm{I}_{\mathbf{M}}, \mathrm{II}_{\mathbf{M}}, \mathrm{III}_{\mathbf{M}}$ of $\mathbf{M}$. In (7.7), $\mathbf{P}$ is a symmetric tensor-valued isotropic function of the symmetric second-order tensor $\mathbf{M}$. The present discussion is concerned with that class of constitutive equations for which $\mathbf{P}$ is an isotropic linear function of $\mathbf{M}$. It follows from Sections 2 to 5 and in particular the second equation in (5.17) that for this class of constitutive equation

$$
\mathbf{P}=\mathrm{b}_{0} \mathbf{I}+\mathbf{M}
$$


where $b_{0}$ is a scalar-valued function of the principal invariants $I_{M}, I_{M}, I I I_{M}$ of $\mathbf{M}$. Substitute the expression for $\mathbf{P}$ given in (7.8) into (7.7) to give the constitutive relation

$$
\mathbf{K}=\beta(\operatorname{tr} \mathbf{M}) \mathbf{I}+2 G \mathbf{M}
$$

where

$$
\beta=2 G \mathrm{~b}_{0} /(\operatorname{tr} \mathbf{M})
$$

is a scalar-valued function of the principal invariants $\mathrm{I}_{\mathbf{M}}, \mathrm{II}_{\mathbf{M}}, \mathrm{III}_{\mathbf{M}}$ of $\mathrm{M}$ and the initial conditions have been taken to be $\mathbf{M}=\mathbf{O}$ when $\mathbf{K}=\mathbf{O}$.

8. The Lode relations. Equation (7.9) can be expressed in deviatoric form:

$$
\mathbf{K}^{\prime}=\delta_{1} \mathbf{S}^{\prime}+\delta_{2}\left(\mathbf{S}^{\prime 2}-\frac{2}{3} \mathbf{S}_{2}^{\prime} \mathbf{I}\right)=2 G\left[\varphi_{1} \mathbf{E}^{\prime}+\varphi_{2}\left(\mathbf{E}^{\prime 2}-\frac{2}{3} \mathbf{E}_{2}^{\prime} \mathbf{I}\right)\right]=2 G \mathbf{M}^{\prime}\left(\mathbf{E}^{\prime}\right)
$$

where use has been made of (4.4) and (7.2). From (8.1), $\mathbf{K}^{\prime 2}=4 G^{2} \mathbf{M}^{\prime 2}$, and hence, with use of (5.3)

$$
\mathrm{K}_{2}^{\prime}=\mathrm{HS}_{2}^{\prime}=4 G^{2} \Psi \mathrm{E}_{2}^{\prime}=4 G^{2} \mathrm{M}_{2}^{\prime}, \quad\left(\mathrm{M}_{2}^{\prime}=\Psi \mathrm{E}_{2}^{\prime}\right) .
$$

The connection between the second principal moments of $\mathbf{M}^{\prime}$ and $\mathbf{E}^{\prime}$, given in the second equation in (8.2), is the counterpart to that for $\mathrm{K}_{2}^{\prime}$ given in (5.3).

(i) First Lode relation: enter the spectral representation of $\mathbf{K}^{\prime}$ from (4.5) into (8.1) to yield

$$
\mathbf{M}^{\prime}=\sum_{r=1}^{3} m_{r}^{\prime} \mathbf{q}_{r} \otimes \mathbf{q}_{r}, \quad \mathbf{E}^{\prime}=\sum_{r=1}^{3} \varepsilon_{r}^{\prime} \mathbf{q}_{r} \otimes \mathbf{q}_{r}
$$

where

$$
m_{i}^{\prime}=k_{i}^{\prime} /(2 G)=\varphi_{1} \varepsilon_{i}^{\prime}+\varphi_{2}\left(\varepsilon_{i}^{\prime 2}-\frac{2}{3} \mathrm{E}_{2}^{\prime}\right)(i=1,2,3), \quad k_{i}^{\prime}=2 G m_{i}^{\prime} .
$$

It follows from the second equation in (3.1), (4.5), and (8.3) that $\mathbf{K}^{\prime}, \mathbf{S}^{\prime}, \mathbf{M}^{\prime}$, and $\mathbf{E}^{\prime}$ have the orthonormal triplet $\mathbf{q}_{i}$ in common and hence $\mathbf{K}^{\prime}, \mathbf{S}^{\prime}, \mathbf{M}^{\prime}$, and $\mathbf{E}^{\prime}$ are coaxial.

Since $\mathbf{K}^{\prime}$ and $\mathbf{M}^{\prime}$ are coaxial, it follows that the Mises circle of radius $r=\left(2 \mathbf{K}_{2}^{\prime}\right)^{1 / 2}$ characteristic of $\mathbf{K}^{\prime}$ is concentric with the Mises circle of radius $r^{*}=\left(2 \mathbf{M}_{2}^{\prime}\right)^{1 / 2}$ characteristic of $\mathbf{M}^{\prime}$. From (8.2)

$$
\mathrm{r}=\left(2 \mathrm{~K}_{2}^{\prime}\right)^{1 / 2}=2 G\left(2 \mathrm{M}_{2}^{\prime}\right)^{1 / 2}=2 G \mathrm{r}^{*} .
$$

Equation (8.5) establishes that $2 G$ scales the Mises circle characteristic of $\mathbf{M}^{\prime}$ into coincidence with the Mises circle characteristic of $\mathbf{K}^{\prime}$.

Substitute the expression for the $k_{i}^{\prime}$ given in (8.4) into (4.10) and use the first equation in (7.5) to give

$$
\chi=\kappa=\text { const. }
$$

The equality given in (8.6) is the first Lode relation.

(ii) Second Lode relation: an alternative geometrical representation of $\mathbf{M}^{\prime}$ is the Prager representation in the form of a regular twelve-sided polygon for which each of the twelve linear sections is of the form given in (6.4); thus

$$
\mathrm{v}^{*}=-3^{1 / 2}\left(\mathrm{u}^{*}-\mathrm{u}_{0}^{*}\right) / \kappa, \quad \mathrm{u}_{0}^{*}=\mathrm{k}^{*}\left[2\left(3+\kappa^{2}\right) / 3\right]^{1 / 2}=\text { const. }
$$


$\operatorname{In}(8.7)$

$$
\mathrm{v}^{*}=\left.(3 / 2)^{1 / 2} \varepsilon_{1}^{\prime}\right|_{\mathbf{M}}, \mathrm{u}^{*}=\left(\varepsilon_{3}^{\prime}-\varepsilon_{2}^{\prime}\right) / \text { bigg } /\left.2^{1 / 2}\right|_{\mathbf{M}}, \kappa=-3^{1 / 2} \mathrm{du}^{*} / \mathrm{dv}^{*}=\left.3^{1 / 2} \tan \phi^{*}\right|_{\mathbf{M}}
$$

where $\phi^{*}$ is the counterpart for $\mathbf{M}$ to $\phi$ defined in the context of (4.11) and (6.5). Since $\mathbf{K}^{\prime}$ and $\mathbf{M}^{\prime}$ are coaxial, it follows that the twelve-sided regular polygon characteristic of $\mathbf{M}^{\prime}$ is concentric with the twelve-sided regular polygon characteristic of $\mathbf{K}^{\prime}$. In (8.8), $\kappa / 3^{1 / 2}$ is the gradient of any arbitrary straight line perpendicular to the linear $\left(\mathrm{u}^{*}, \mathrm{v}^{*}\right)$ relation given in the first equation in (8.7).

The first Lode relation, together with (6.5) and the third equation in (8.8) give

$$
\chi=-3^{1 / 2} \mathrm{du} / \mathrm{dv}=\left.3^{1 / 2} \tan \phi\right|_{\mathbf{K}}=-3^{1 / 2} \mathrm{du}^{*} / \mathrm{dv}^{*}=\left.3^{1 / 2} \tan \phi^{*}\right|_{\mathbf{M}}=\kappa=\text { const. }
$$

From $(8.9)$ it is seen for $\mu:[0, \infty], \chi:[0, \infty]$ that the first Lode relation gives $\left.\phi\right|_{\mathbf{K}}=\left.\phi^{*}\right|_{\mathbf{M}}$. This condition establishes that these two twelve-sided regular polygons are geometrically identical: that is, within each of the twelve $\pi / 6$ sections, the two corresponding linear sections of the regular twelve-sided polygons form a pair of parallel lines.

In the context of the discussion given in Section 6, in particular (6.6) and (6.1), it follows that for $\nu=\kappa$, the line defined by the linear $\left(\mathrm{u}^{*}, \mathrm{v}^{*}\right)$ relation given in the first equation in (8.7) is a tangent to the Mises circle of radius $R^{*}=\left(2 E_{2}^{\prime}\right)^{1 / 2}$ characteristic of the geometrical representation of $\mathbf{E}^{\prime}$ in the deviatoric plane.

For the particular condition $\mu_{0}=\chi_{0}$, let a linear section of the regular twelve-sided polygon characteristic of $\mathbf{K}^{\prime}$ be a tangent at the point $\mathrm{P}(\mathrm{u}, \mathrm{v})$ to the Mises circle of radius R. Similarly, for the particular condition $\nu_{0}=\kappa_{0}$, let the corresponding linear section of the regular twelve-sided polygon characteristic of $\mathbf{M}^{\prime}$ be a tangent at the point $\mathrm{Q}\left(\mathrm{u}^{*}\right.$, $\left.\mathrm{v}^{*}\right)$ to the Mises circle of radius $\mathrm{R}^{*}$. The first Lode relation, that is $\chi=\kappa$, identifies for the particular conditions $\mu_{0}=\chi_{0}$ at $\mathrm{P}(\mathrm{u}, \mathrm{v})$ and $\nu_{0}=\kappa_{0}$ at $\mathrm{Q}\left(\mathrm{u}^{*}, \mathrm{v}^{*}\right)$ that

$$
\left[\chi_{0}=\left.3^{1 / 2} \tan \phi\right|_{\mathbf{K}}=\mu_{0}=3^{1 / 2} \tan \alpha_{0}=\kappa_{0}=\left.3^{1 / 2} \tan \phi^{*}\right|_{\mathbf{M}}=\nu_{0}=3^{1 / 2} \tan \alpha_{0}^{*}\right]_{\mathrm{P}, \mathrm{Q}}
$$

where use has been made of $(2.6),(6.5)$ and the third equation in (8.8). In (8.10), the expression $\nu_{0}=3^{1 / 2} \tan \alpha_{0}^{*}$ is the counterpart for $\mathbf{M}^{\prime}$ to the expression for $\mu$ given in (2.6). Equation (8.10) establishes (i) that the points $\mathrm{P}$ and $\mathrm{Q}$ must both be on the same straight line in the deviatoric plane through the origin o of the system $(o, q)$, and (ii) that the line oPQ is characterised by the condition $\mu_{0}=\chi_{0}=\kappa_{0}=\nu_{0}$ and hence (5.9) and (7.4) give

$$
[\mathrm{H}]_{\mu_{0}=\chi_{0}}=1, \quad[\Psi]_{\nu_{0}=\kappa_{0}}=1, \quad\left[\mu_{0}=\chi_{0}=\kappa_{0}=\nu_{0}\right]_{\mathrm{P}, \mathrm{Q}} .
$$

Equation (8.2) can be used, together with (2.1), (2.4), (7.5), (7.6), (8.2), (8.10), and (8.11), to give for the corresponding points $\mathrm{P}$ and $\mathrm{Q}$

$$
\left.\left(s_{3}^{\prime}-s_{2}^{\prime}\right)\right|_{\mathbf{K}}=\left.2 G\left(\varepsilon_{3}^{\prime}-\varepsilon_{2}^{\prime}\right)\right|_{\mathbf{M}},\left.\quad s_{1}^{\prime}\right|_{\mathbf{K}}=\left.2 G \varepsilon_{1}^{\prime}\right|_{\mathbf{M}},\left[\mu_{0}=\chi_{0}=\kappa_{0}=\nu_{0}\right]_{\mathrm{P}, \mathrm{Q}} .
$$

Equations (6.1), (8.8), and (8.12) yield

$$
\left.\mathrm{u}\right|_{\mathbf{K}}=\left.2 G \mathrm{u}^{*}\right|_{\mathbf{M}},\left.\quad \mathrm{v}\right|_{\mathbf{K}}=\left.2 G \mathrm{v}^{*}\right|_{\mathbf{M}}, \quad\left[\mu_{0}=\chi_{0}=\kappa_{0}=\nu_{0}\right]_{\mathrm{P}, \mathrm{Q}} .
$$

The conditions established in the context of (8.7)-(8.13) apply in all twelve $\pi / 6$ sections. 
Equation (8.13) establishes that $2 G$ scales the twelve-sided regular polygon characteristic of $\mathbf{M}^{\prime}$ into coincidence with the twelve-sided regular polygon characteristic of $\mathbf{K}^{\prime}$. From this it follows that $2 G$ scales the Mises circle characteristic of $\mathbf{E}^{\prime}$ into coincidence with the Mises circle characteristic of $\mathbf{S}^{\prime}$ and hence

$$
\mathrm{R}=\left(2 \mathrm{~S}_{2}^{\prime}\right)^{1 / 2}=2 G\left(2 \mathrm{E}_{2}^{\prime}\right)^{1 / 2}=2 G \mathrm{R}^{*} .
$$

From (3.5), (8.2), and (8.14)

$$
\mathrm{H}\left(2 \mathrm{~S}_{2}^{\prime}\right)=\mathrm{HR}^{2}=\Psi 4 G^{2}\left(2 \mathrm{E}_{2}^{\prime}\right)=\Psi 4 G^{2} \mathrm{R}^{* 2} .
$$

Substitute the expression for $\mathrm{R}$ given in (8.14) into (8.15) to give the condition

$$
\mathrm{H}=\Psi
$$

for all $\mu$ and all $\nu$. Equation (8.16) together with the use of the first Lode relation and equations (5.9) and (7.4) gives

$$
\left[(\mu+\nu) \chi^{2}+2(3-\mu \nu) \chi-3(\mu+\nu)\right](\mu-\nu)=0 .
$$

The only solution of (8.17) valid for all $\mu$ and all $\nu$ is

$$
\mu=\nu \text {. }
$$

The equality given in (8.18) is the second Lode relation.

Changes in the magnitude of $\mathbf{S}$, that is, variation of $\left(S_{2}^{\prime}\right)^{1 / 2}$, leads either to expansion or contraction of the area of the twelve-sided regular polygon characteristic of $\mathbf{K}^{\prime}$. Any variation in the loading of $S_{2}^{\prime}$ leads to a constitutive variation in $M_{2}^{\prime}$ in the form of either an expansion or a contraction of the area of the twelve-sided regular polygon characteristic of $\mathbf{M}^{\prime}$. Central to the formulation of a constitutive equation is the concept that $2 G$ scales the two polygons into coincidence, which is only possible if $\mu=\nu$ for all $\mu$ and all $\nu$. It is in this latter context that the second Lode relation, given in $(8.18)$, is central to nonlinear constitutive theory.

9. Constitutive equation. In the context of constitutive theory, the characteristic representation intensity function, in the form defined on the right-hand side in (5.4) is effectively a criterion for determining admissible systems of loading, and as such must be retained in any general form of constitutive equation. Substitute in (8.2) the expression for $S_{2}^{\prime}$ given in (2.4) and the expression for $E_{2}^{\prime}$ given in (7.6) to give, with use of the second Lode relation,

$$
\mathrm{H}^{1 / 2}\left(s_{3}^{\prime}-s_{2}^{\prime}\right)=2 G \Psi^{1 / 2}\left(\varepsilon_{3}^{\prime}-\varepsilon_{2}^{\prime}\right)
$$

From (9.1), (2.1), and the second equation in (7.5), together with the use of (3.2) and the second Lode relation, it follows that

$$
\mathrm{H}^{1 / 2} s_{1}^{\prime}=-\mathrm{H}^{1 / 2}\left(s_{2}^{\prime}+s_{3}^{\prime}\right)=2 G \Psi^{1 / 2} \varepsilon_{1}^{\prime}=-2 G \Psi^{1 / 2}\left(\varepsilon_{2}^{\prime}+\varepsilon_{3}^{\prime}\right) .
$$

Equations (9.1) and (9.2), together with use of the second Lode relation, yield

$$
\mathrm{H}^{1 / 2} s_{2}^{\prime}=2 G \Psi^{1 / 2} \varepsilon_{2}^{\prime}, \quad \mathrm{H}^{1 / 2} s_{3}^{\prime}=2 G \Psi^{1 / 2} \varepsilon_{3}^{\prime} .
$$


Enter the expressions given in (9.2) and (9.3) for the $s_{i}^{\prime}(i=1,2,3)$ into the second equation in (3.1) and use (8.3) to establish

$$
\mathrm{H}^{1 / 2} \mathbf{S}^{\prime}=\mathrm{H}^{1 / 2}\left[\mathbf{S}-\frac{1}{3}(\operatorname{tr} \mathbf{S}) \mathbf{I}\right]=2 G \Psi^{1 / 2} \mathbf{E}^{\prime}=2 G \Psi^{1 / 2}\left[\mathbf{E}-\frac{1}{3}(\operatorname{tr} \mathbf{E}) \mathbf{I}\right] .
$$

It has been shown in Section 8.(ii) that $\mathrm{H}=\Psi$ for all $\mu$ and for all $\nu$.

Equation (9.4) can be rearranged to give the constitutive equation

$$
\boldsymbol{\Theta}=\lambda(\operatorname{tr} \boldsymbol{\Phi}) \mathbf{I}+2 G \boldsymbol{\Phi},
$$

where the initial conditions have been taken to be $\mathbf{E}=\mathbf{O}$ when $\mathbf{S}=\mathbf{O}$, and

$$
\Theta=\mathrm{H}^{1 / 2} \mathbf{S}, \quad \boldsymbol{\Phi}=\Psi^{1 / 2} \mathbf{E}
$$

are generalized constitutive state variables. The retention of the Prager loading and response parameters in (9.4) and (9.5) ensures an implicit dependence of the constitutive equation upon the characteristic representation intensity function.

The nonlinear response of $\Phi$ to changes in the loading of $\Theta$ is described by the coefficient $G$ through its dependence upon the principal invariants $\mathrm{I}_{\mathbf{E}}, \mathrm{II}_{\mathbf{E}}, \mathrm{III}_{\mathbf{E}}$ of $\mathbf{E}$. There are as many observable characteristic constitutive relations as there are individual admissible systems of loading.

For those elastic materials for which $\chi=\mu, \kappa=\nu$ for all $\mu(=\nu)$, it follows from (5.9) that $\mathrm{H}=1$ and from (7.4) that $\Psi=1$; these conditions reduce (9.5) to the constitutive equation given in (1.1).

10. Concluding remarks. The properties characteristic of the representation theorem for isotropic tensor-valued functions of symmetric second-order tensors are determined by two parameters referred to as the Lode parameter, which is continuously variable, and the Lode representation number. The range of application of the representation theorem is determined by the values of the Lode representation number.

A geometrical representation has been established by way of the concept of a characteristic representation intensity function. The geometrical representation identifies the only admissible form of the representation intensity function to be piecewise linear and continuous.

These properties of the representation theorem limit the way in which this theorem can be used in the formulation of constitutive equations.

The representation theorem has been used to formulate a generalisation of Hooke's law for finite strain applicable to the initial elastic range of strain-hardening materials, including the elastic conditions at initial yield.

\section{ReFERENCES}

[1] E. W. Billington, Constitutive equation for a class of isotropic perfectly elastic solids using a new measure of finite strain and the corresponding stress, J. Eng. Math. 45. 117-134 (2003)

[2] P. G. Ciarlet, Mathematical Elasticity, Volume I, Three dimensional elasticity. North Holland: Amsterdam 1988, pp. 115-118, 130-132

[3] H. Fromm, Stoffgesetze des isotropen Kontinuums, insbesondere bei Zähplastischem Vehalton, IngArch 4. 432-466 (1933)

[4] A. M. Goodbody, Cartesian tensors, Ellis Horwood: Chichester, 1982 pp. 106-108

[5] W. Lode, Versuche uber den einfluss der mittleren hauptspannung auf das fliessen der metalle eisen, kupfer und nickel, Z. Phys. 36. 913-939 (1926) 
[6] R. von Mises, Mechanik, der festen Körper in plastich deformablen Zustand, Göttinger Nachrichten. Math.-Phys Klasse 582-592 (1913)

[7] R. von Mises, Mechanik der plastischen Formänderung von Kristallen, ZAMM 8. 161-185 (1928)

[8] J. H. Poynting, On pressure perpendicular to the shear-planes in finite pure shear and on the lengthening of loaded wires when twisted, Proc. Roy. Soc.(London) A82. 546-559 (1909)

[9] J. H. Poynting, On the changes in the dimensions of a steel wire when twisted, and on the pressure of distortional waves in steel, Proc. Roy. Soc.(London) A86. 534-561 (1912)

[10] W. Prager, Strain hardening under combined stresses, J. App. Phys. 16. 837-840 (1945). 